\title{
The Wandering Foreign Body: A Seemingly Spontaneous Migration from Bronchus to Stomach in a Child
}

\author{
Richard Lambert ${ }^{1}$ \\ ${ }^{1}$ Geisinger Medical Center, Janet Weis Children's Hospital, Danville, \\ Pennsylvania, United States
}

J Child Sci 2021;11:e68-e69.

A 12-year-old previously healthy male presented to a referring emergency department (ED) after reporting that he suddenly "breathed in a piece of a toy" while riding in a car. The child had removed a metal pin from a "fidget cube" and was chewing on it. The car was jarred as it drove over a bump, leading him to inhale the metal pin.

In the ED, a chest radiograph obtained around 2000 revealed a cylindrical, radiopaque foreign body (FB) in his right bronchus ( - Fig. 1). The child denied shortness of breath,

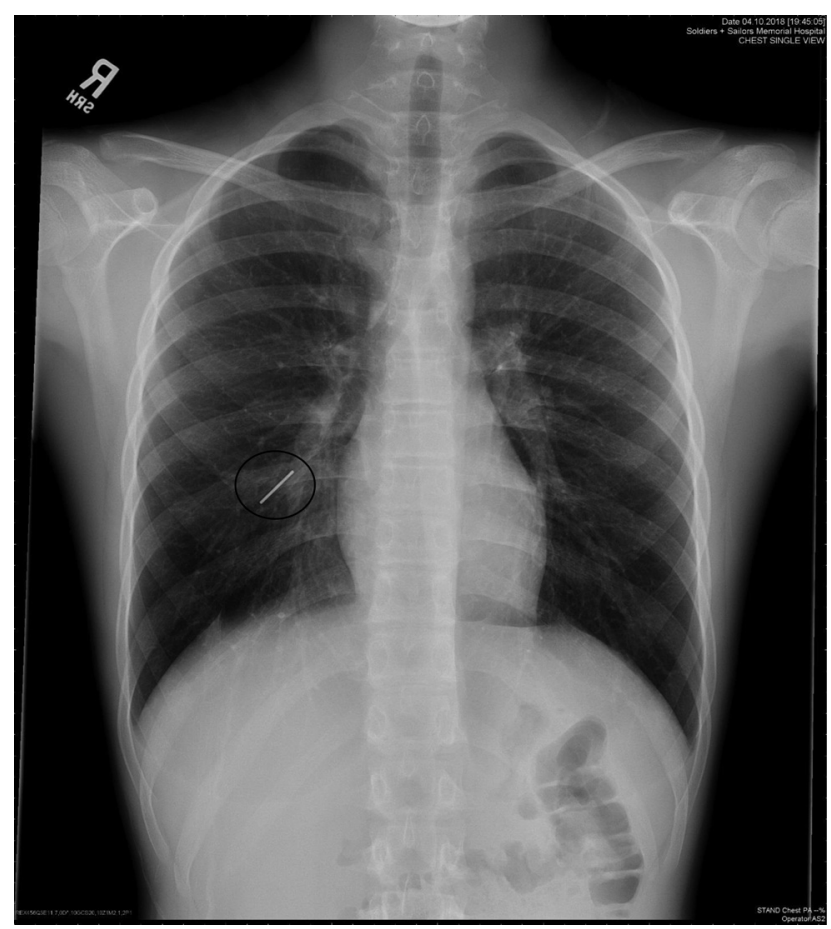

Fig. 1 Foreign bodies in right bronchus.

DOI https://doi.org/ $10.1055 / \mathrm{s}-0041-1726098$ ISSN 2474-5871.
Address for correspondence Richard Lambert, Geisinger Medical Center, Janet Weis Children's Hospital, Danville, Pennsylvania, United States (e-mail: rllambert@geisinger.edu).

chest pain, or any discomfort. He had no coughing or vomiting. His vital signs were stable, and his oxygen saturation was 99\% on room air. The child was transferred to our pediatric intensive care unit (PICU) for close monitoring and further evaluation by the pediatric otolaryngology staff.

Upon arrival to the PICU, the child remained without distress and reported no coughing or vomiting during transport. The child was monitored overnight and taken to the operating room early the next morning. Under general endotracheal anesthesia, a 13.5 slotted Storz laryngoscope was used to evaluate the pharynx, larynx, and periglottic regions and no abnormalities were noted. A long Hopkins telescope was then used to examine the trachea and both right and left mainstem bronchi. No FB or signs of inflammation were identified. An intraoperative chest radiograph obtained around 0900 revealed that the FB was now in the stomach (-Fig. 2). The pediatric gastroenterology service was consulted and suggested that the FB did not require removal, as it posed no threat to his digestive system

Foreign bodies in either the pulmonary or gastrointestinal tract are not uncommon in children. ${ }^{1}$ Spontaneous expectoration of a FB with subsequent migration has been reported previously in children less than 5 years of age. ${ }^{2,3}$ In these cases, the inciting event was not witnessed, and the children did not present with symptoms for weeks to months after aspiration. Retention of a FB can lead to lung atelectasis, infection, or abscess formation. If the $\mathrm{FB}$ is radiolucent, it may not be seen on the radiograph, but can be detected with conventional computed tomography ${ }^{4}$ or multidetector-row computed tomography, also known as virtual bronchoscopy. ${ }^{5}$

In our case, the initial radiograph clearly revealed that the $\mathrm{FB}$ was in the right bronchus. In the operating suite, the subsequent imaging revealed the FB to be in the stomach. Other authors have reported stressful coughing as the

\section{(c) 2021. The Author(s)}

This is an open access article published by Thieme under the terms of the Creative Commons Attribution License, permitting unrestricted use, distribution, and reproduction so long as the original work is properly cited. (https://creativecommons.org/licenses/by/4.0/)

Georg Thieme Verlag KG, Rüdigerstraße 14, 70469 Stuttgart, Germany 


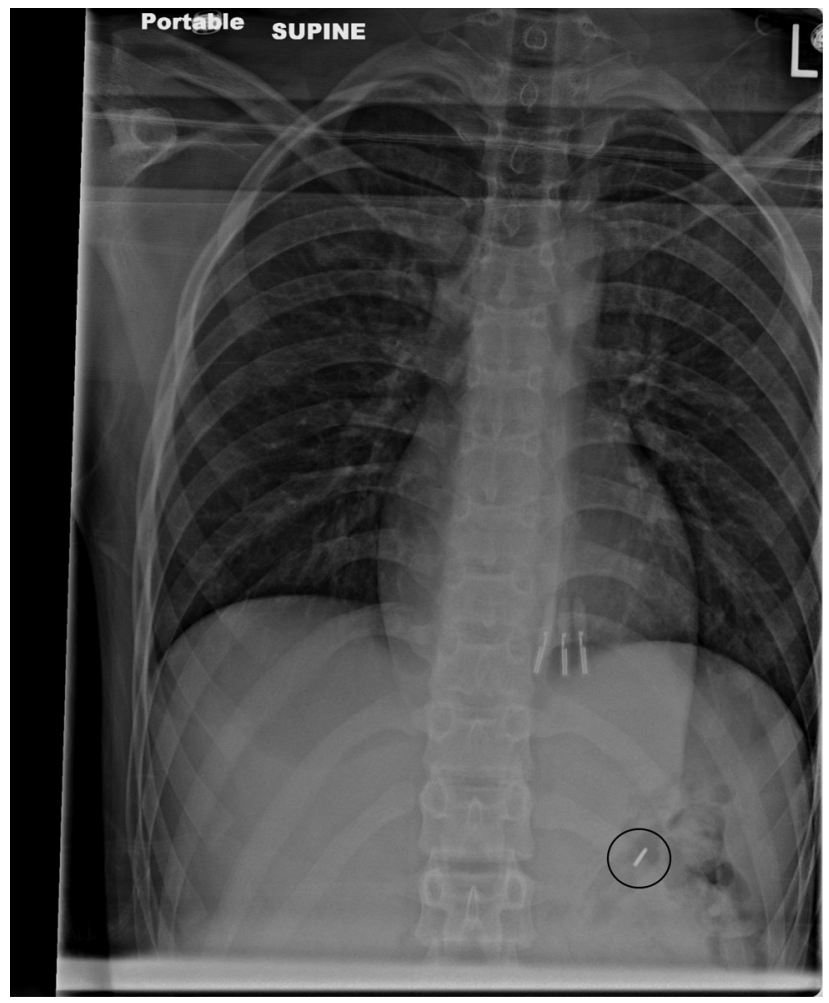

Fig. 2 Foreign body in stomach.

mechanism for a peanut migrating from the right bronchus to the left bronchus. ${ }^{6}$ Our child denied coughing and there were no witnessed coughing episodes to explain the FB moving from the bronchus to the stomach. Nonetheless, we propose that the only explanation is the child must have coughed up the FB into his oropharynx and then swallowed it into his digestive tract. If a chest radiograph had been obtained prior to the child undergoing general anesthesia and rigid bronchoscopy in the operating suite, significant costs and potential morbidity could have been avoided.

Conflict of Interest

None declared.

\section{References}

1 Rothmann BF, Boeckman CR. Foreign bodies in the larynx and tracheobronchial tree in children. A review of 225 cases. Ann Otol Rhinol Laryngol 1980;89(5 Pt 1):434-436

2 Singh RB, Gangopadhyay AN, Gupta DK, Pandey V. Migrating foreign body bronchus: an unusual case of foreign body aspiration. Case Rep Clin Med 2014;3:407-409

3 Aihole JS, Babu MN. Spontaneous migration of airway foreign body to the gastrointestinal tract. Indian Pediatr 2015;52(06): 534-535

4 Berger PE, Kuhn JP, Kuhns LR. Computed tomography and the occult tracheobronchial foreign body. Radiology 1980;134(01): 133-135

5 Sodhi KS, Saxena AK, Singh M, Rao KL, Khandelwal N. CT virtual bronchoscopy: new non invasive tool in pediatric patients with foreign body aspiration. Indian $\mathrm{J}$ Pediatr 2008;75(05):511-513

6 Wu CT, Wang CJ. Alternate lung collapse in a 9-year-old boy with peanut aspiration. Pediatr Radiol 2006;36(12):1327-1328 